\title{
Molecular packing of non-fullerene acceptors for organic solar cells: Distinctive local morphology in Y6 versus ITIC derivatives
}

\author{
Grit Kupgan, Xian-Kai Chen, and Jean-Luc Brédas* \\ Department of Chemistry and Biochemistry \\ The University of Arizona \\ Tucson, Arizona 85721-0088, United States
}

*Correspondence: jlbredas@arizona.edu (J.L. Brédas) 


\begin{abstract}
Since a couple of years ago, Y6 has emerged as one of the main non-fullerene acceptors for organic solar cells as its use leads to superior power conversion efficiencies. It is thus of major interest to investigate the multi-scale phenomena that are responsible for Y6's efficacy. Here, we modeled neat films of Y6 and earlier non-fullerene acceptors, IT-4F and ITIC, using a combination of density functional theory calculations and molecular dynamics simulations, to investigate the various factors that control their charge and exciton transport rates. We find that the molecular packing in Y6 is drastically different from that in IT-4F and ITIC. At the nano-scale, the local morphology of Y6 consists of a large number of directional face-on stackings and well-connected transport networks. Y6 also consistently shows higher electronic couplings for LUMOs, HOMOs, and local excitations than ITIC-type acceptors, which results in fast transport rates for electron, holes, and excitons. Importantly, when considering dimers, their configurations in Y6 are more diverse than in ITIC-type acceptors, with many of those similar to the configurations observed in the Y6 crystal structure reported recently. Most Y6 dimer configurations exhibit strong binding interactions, large electronic couplings, and high transport rates, which when taken together rationalize the better performance of OSCs based on Y6.
\end{abstract}

Keywords: Organic solar cells, non-fullerene acceptors, molecular packing, density functional theory, molecular dynamics. 


\section{Introduction}

Organic solar cells (OSCs) can be a promising solution for the delivery of clean and renewable energy in a number of applications [1-4]. The benefits of using organic materials have been widely discussed, which include light weight, flexibility, desirable mechanical properties, and scalability [1-4]. The newer generations of organic solar cells are based on non-fullerene acceptors (NFAs) [5-8], which consist of fused-ring structures built from donor (D) and acceptor (A) moieties. Due to the modularity of the design, chemical modifications (e.g., fluorination, chlorination, addition of alkoxy groups, side-chain modulation) are widely used to tune energy levels, solubility, and morphology in order to maximize the power conversion efficiency (PCE) [6-8]. The first wave of NFA's was based on an A-D-A molecule referred to as ITIC and developed by Zhan and coworkers in 2015 [5]. The PCEs of OSCs with ITIC-type acceptors quickly rose from $6.8 \%$ to 13\% [9], making a significant improvement over traditional fullerene-based acceptor devices [10-13]. A second wave of NFA's emerged with the development of the so-called Y-type acceptors [14-16], which have an A-DA'D-A structure, and especially of Y6 in 2019, which resulted at the time in an unprecedented PCE of $\sim 15 \%$ [17]. The record PCE of Y6-based single-junction OSCs is currently $\sim 18 \%[18,19]$.

There are major on-going efforts not only to optimize blends containing Y6 or Y6 derivatives, but also to elucidate the mechanisms behind their remarkable properties [20-23]. In terms of charge transport, Y-type acceptors can generally achieve higher mobilities than ITIC-type acceptors. However, the fundamental rationale behind this observation is difficult to probe in thin films due to the complex nature of the local and global morphologies. Thus, one approach is to investigate the differences in molecular packing via single-crystal X-ray diffraction [24]. Although thin films of these non-fullerene acceptors are not entirely crystalline, such an approach can be useful as 
some degree of crystallinity is retained after processing [25]. From the analysis of crystal structures, unlike most ITIC-type acceptors, Y6 acceptors typically contain three-dimensional interpenetrating networks, which enable better transport properties by unlocking multiple pathways [26-29]. From the crystal structure of Y-type acceptors, the configurations of dimers revealed a combination of H-type and J-type aggregates (through interactions between cores and terminal moieties), while ITIC-type acceptors predominantly contain J-type aggregates (via terminal-terminal interactions) [30]. Among the different configurations in the crystal, our earlier quantum-mechanical calculations indicated that these packings lead to various degrees of electronic couplings for electrons, holes, and excitons [25].

In this work, we focus on the amorphous regions within the thin films of Y6 and ITIC-type acceptors (Figure 1). We note that probing the local morphology within the amorphous phase is non-trivial because experimental techniques cannot provide a complete picture of the local (that is, nano-scale) morphology. Therefore, to gain high-resolution molecular packing information, we employed a combination of long-range corrected density functional theory (DFT) calculations and molecular dynamics (MD) simulations, which have been essential in relating structures, electronic properties, and OSC performance [31,32]. Recently, such as combined DFT/MD approach has been used to investigate the donor/acceptor interfacial properties of Y6-based OSCs to uncover their effects on exciton-dissociation and charge generation processes [33-36]. Here, we construct neat films of Y6, IT-4F, and ITIC via MD simulations in order to represent the amorphous phases of the acceptor domains within the active layers in OSCs. Based on the local morphology obtained from these MD simulations, we make the comparison between the three acceptors in terms of molecular packing, network extensivity, electronic properties, and transport rates. Also, we 
investigate how the various packing configurations in Y6 influence intermolecular interaction energies, electronic properties, and transport rates for electrons, holes, and excitons.
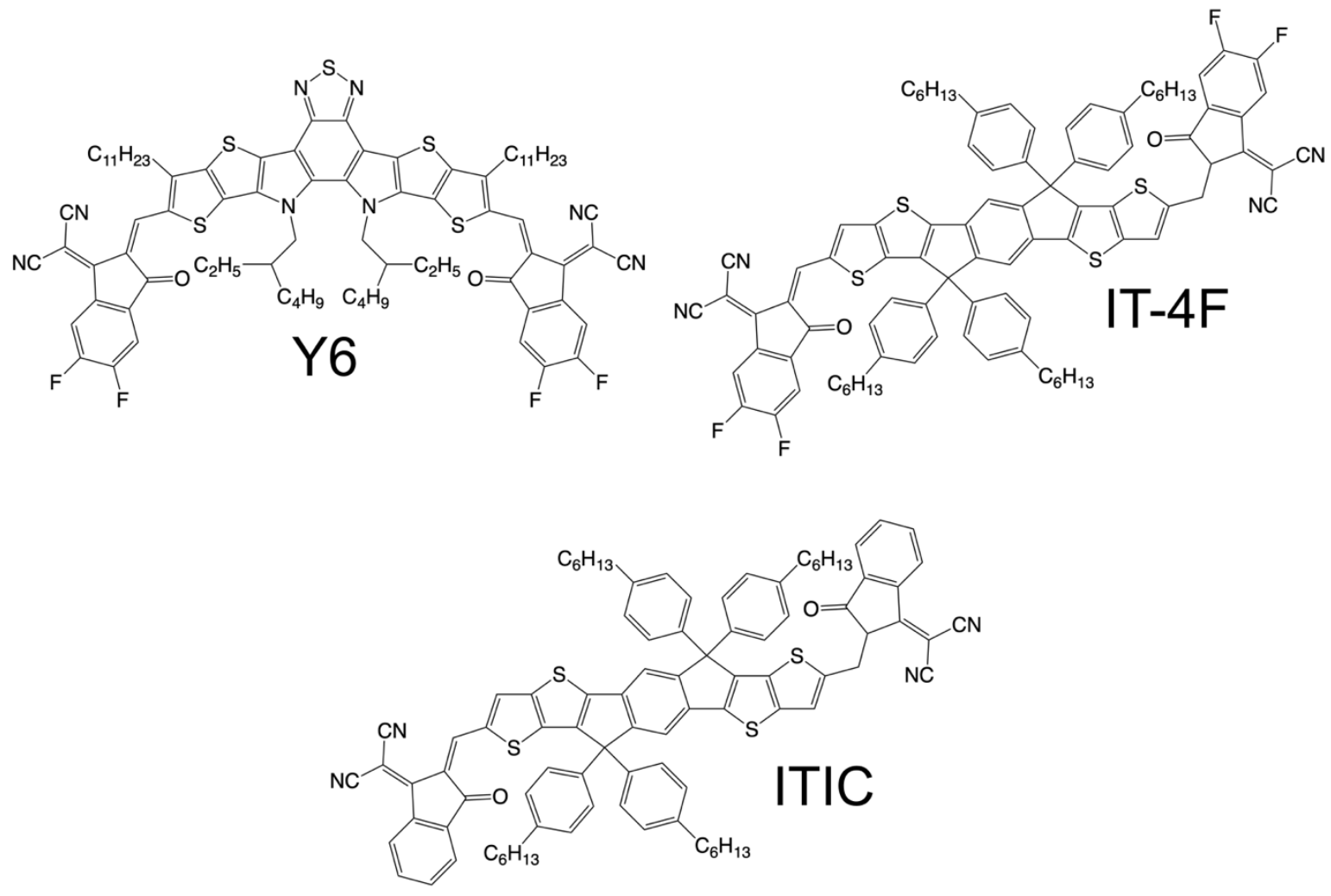

Figure 1. The non-fullerene electron acceptors (NFAs) investigated in this work: Y6, IT-4F, and ITIC. $\quad \mathrm{Y} 6$ is 2,2'-((2Z,2'Z)-((12,13-bis(2-ethylhexyl)-3,9-diundecyl-12,13-dihydro[1,2,5]thiadiazolo[3,4-e]thieno[2", $\left.3^{\prime \prime}: 4^{\prime}, 5^{\prime}\right]$ thieno[2',3':4,5]pyrrolo[3,2-

g]thieno[2',3':4,5]thieno[3,2-b]indole-2,10-diyl)bis(methanylylidene))bis(5,6-difluoro-3-oxo-2,3dihydro-1H-indene-2,1-diylidene))dimalononitrile; IT-4F is 3,9-bis(2-methylene-((3-(1,1dicyanomethylene)-6,7-difluoro)-indanone))-5,5,11,11-tetrakis(4-hexylphenyl)-dithieno[2,3d:2',3'-d']-s-indaceno[1,2-b:5,6-b']dithiophene; and ITIC is 3,9-bis(2-methylene-(3-(1,1dicyanomethylene)-indanone))-5,5,11,11-tetrakis(4-hexylphenyl)-dithieno[2,3-d:2',3'-d']-sindaceno[1,2-b:5,6-b']dithiophene. 


\section{Computational methods}

\subsection{Molecular dynamics simulations}

Neat films of Y6, IT-4F, and ITIC were constructed using all-atom molecular dynamics simulations performed in LAMMPS [37]. The non-bonded and bonded energies were based on the OPLS-AA force field [38-40] with a few modifications as follows. The partial charges for these acceptors were based on the results of long-range corrected $\omega \mathrm{B} 97 \mathrm{XD}$ [41] calculations with the cc-PVTZ basis set; they were fitted using the restrained electrostatic potential method in AmberTools 18 [42]. The equilibrium bond lengths and bond angles were updated based on the DFT $(\omega \mathrm{B} 97 \mathrm{XD} / 6-31 \mathrm{G}(\mathrm{d}, \mathrm{p}))$ optimized geometries. The dihedral potentials between the core and the terminal group were also updated based on the DFT $(\omega \mathrm{B} 97 \mathrm{XD} / 6-31 \mathrm{G}(\mathrm{d}, \mathrm{p}))$ scan of potential energy surfaces. All of the DFT calculations were performed using Gaussian 16 [43]. The optimized force-field parameters are provided at the end of the Supplementary Information.

The neat films were obtained from several stages of isothermal-isobaric ensemble molecular dynamics (NPT-MD). First, 200 molecules of acceptors were randomly placed in an empty cubic simulation box with periodic boundary conditions at low density $\left(\sim 0.1 \mathrm{~g} / \mathrm{cm}^{3}\right)$ using the code from Polymatic [44]. Following the random placement of molecules, a 30 ns of NPT-MD was performed at $650 \mathrm{~K}$ and $1 \mathrm{~atm}$ to equilibrate the system. Afterwards, the films were cooled at 1 atm from 650 $\mathrm{K}$ to $300 \mathrm{~K}$ at a rate of $10 \mathrm{~K} / \mathrm{ns}$. Then, another $30 \mathrm{~ns}$ of NPT-MD was performed at $300 \mathrm{~K}$ and 1 atm to obtain the amorphous films. All of the results were analyzed from the last $10 \mathrm{~ns}$ of the NPTMD. The velocity-Verlet algorithm with 2 fs timestep and SHAKE algorithm were used in all MD simulations. The pressure and temperature were controlled with the Nose-Hoover barostat and thermostat. The non-bonded interactions employed $12 \AA$ cutoff with particle-particle particle-mesh (PPPM) for the electrostatics term. A total of 3 independent simulation boxes for each acceptor 
were constructed from scratch to account for variations in bulk structures. For subsequent DFT calculations, monomers and dimers were extracted from snapshots of the MD simulations using the Pysimm tool [45]. For dimers, our extraction criterion is based on the number of "contacts" between adjacent molecules, where each contact refers to an inter-atomic distance of less than $4 \AA$ in a dimer.

\subsection{Electronic-structure calculations}

The electron affinities (EA), ionization potentials (IP), lowest-excited singlet energies, and reorganization energies of these acceptors were calculated with long-range corrected DFT and TDDFT $(\omega \mathrm{B} 97 \mathrm{XD} / 6-31 \mathrm{G}(\mathrm{d}, \mathrm{p}))$. The $\omega$ parameters were non-empirically tuned by minimizing the differences between the highest occupied [lowest unoccupied] molecular orbital energy ( $\mathrm{E}_{\text {номо }}$ ) $\left[\left(E_{\mathrm{LUMO}}\right)\right]$ and IP $[\mathrm{EA}]$, under an implicit dielectric environment based on the polarizable continuum model (PCM) (taking $\varepsilon=3.0$ as a typical value for organic semiconductors) [46]. The tuned $\omega$ values for Y6, IT-4F, and ITIC are $0.0126,0.0125$, and $0.0125 \mathrm{Bohr}^{-1}$, respectively.

The electronic coupling (V) between two molecules in a dimer was calculated based on the fragment orbital approach [47]. The couplings between LUMO-LUMO [HOMO-HOMO] levels were used for electron [hole] transport rate calculations. For excitons, the coupling was evaluated via the electronic energy transfer method in Gaussian 16 [43].

The charge transfer rates between two molecules in a dimer were calculated based on the semiclassical Marcus equation [48]:

$$
\mathrm{k}=\frac{2 \pi}{\hbar} \frac{|\mathrm{V}|^{2}}{\sqrt{4 \pi \lambda \mathrm{k}_{\mathrm{B}} \mathrm{T}}} \exp \left[-\frac{(\Delta \mathrm{E}+\lambda)^{2}}{4 \lambda \mathrm{k}_{\mathrm{B}} \mathrm{T}}\right]
$$


where $\mathrm{V}$ denotes the electronic coupling; $\lambda$, the reorganization energy; $\Delta \mathrm{E}$, the energy difference between the initial and final states (i.e., differences in EA, IP, or lowest excited singlet state energy between molecules in a selected dimer); and $\mathrm{T}$, the temperature. The intramolecular reorganization energies for electrons $\left(\lambda_{\mathrm{e}}\right)$, holes $\left(\lambda_{\mathrm{h}}\right)$, and excitons $\left(\lambda_{\mathrm{x}}\right)$ were calculated based on adiabatic potential energy surfaces [47]; these values are reported in Table S1. Since the intermolecular reorganization energy is not straightforward to evaluate, it was set at $0.1 \mathrm{eV}$, a reasonable value for extended $\pi$-conjugated systems $[49,50]$.

The decomposition of molecular interactions between pairs of acceptor molecules was evaluated via the symmetry-adapted perturbation theory (SAPT) code in Psi4 [51, 52]. The SAPT approach allows us to deconstruct molecular interactions into physically meaningful components including electrostatic, exchange-repulsion, induction, and dispersion terms, which govern the overall interaction energy. We employed SAPT with the simplest truncation (SAPT0) and the 6-31G(d,p) basis set; the self-consistent field procedure was based on the density-fitted algorithm along with initial guess from a superposition of atomic densities. The core orbitals were frozen during the SAPT calculations. The limitation of 10 pairs per configuration is due to the high computational cost of wavefunction-based SAPT0 calculations. In our previous work, we have demonstrated that the total interaction energies from SAPT0 were fully consistent with counterpoise-corrected interaction energies obtained from long-range corrected DFT [53]. 


\section{Results and discussion}

\subsection{Local morphologies}

It is well-established that the molecular packings directly influence the transport properties [54, 55]. Thus, it is of major interest to consider the differences in local morphology among these acceptors. A simple visual inspection of Figure 2 underlines that the Y6 local morphology is markedly different from those of IT-4F and ITIC. The Y6 films contain a considerable amount of directional stacking of multiple molecules, while IT-4F and ITIC appear to have a much more amorphous nature. The radial distribution function data from our previous work showed that the Y6 molecules can interact at the level of their core moieties which enables the observed stacking arrangement [25]. Note that such types of interaction involving the core moieties is prevented in ITIC-type acceptors due to the presence of their out-of-plane hexylphenyl side-chains [56]. The Y6 molecule itself also shows a higher rigidity than ITIC-type acceptors based on the potential energy scan of the dihedral angles between the terminal and core moieties (Figure S1). Thus, the Y6 molecules in the MD-simulated neat films adopt more co-planar conformations than ITIC-type acceptors, as quantified by the dihedral-angle distributions. 


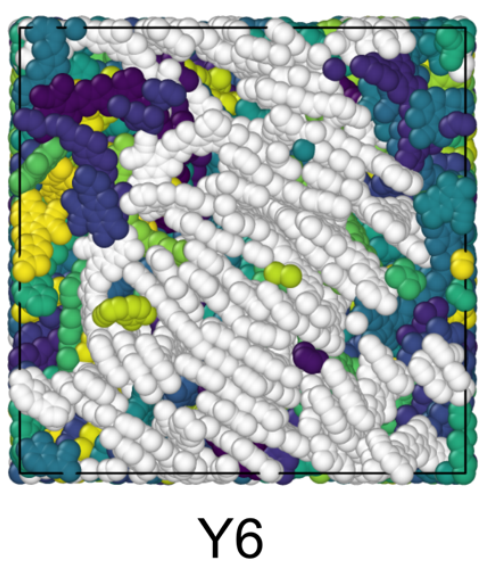

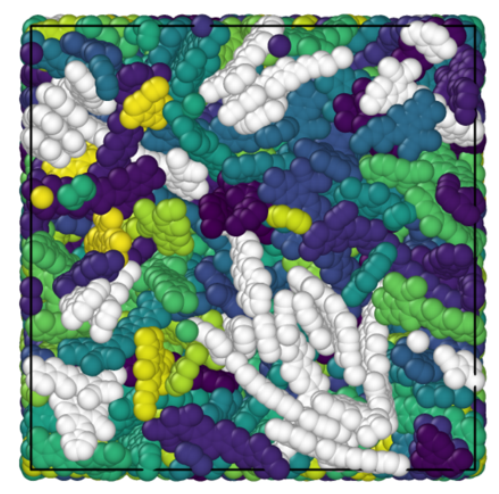

IT-4F

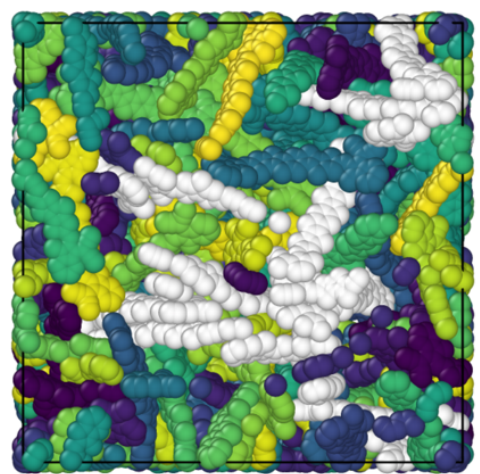

ITIC

Figure 2. Visualization of the overall packing from MD simulations on representative samples. The side chains were removed to improve clarity. Clusters of directional stackings are shown in white; other colors represent individual acceptor molecules.

To characterize the local morphology in our samples, we evaluated the amount of face-on interactions based on dimers extracted from our MD simulations. The total numbers of dimers were obtained by extracting every pair of molecules with at least one contact (as defined in the Computational methods section). Dimers with at least 25 contacts tend to have a face-on configuration; thus, we labeled them as such. Based on our data (Table 1), the Y6 films contain nearly double the amount of face-on interactions relative to IT-4F and ITIC.

Table 1. Quantification of total pairs and face-on pairs found in the MD simulations of Y6, IT-4F, and ITIC neat films. The statistical errors represent variations over 3 independent MD boxes.

\begin{tabular}{ccc}
\hline & Total pairs & Pairs with face-on \\
\hline Y6 & $576 \pm 5$ & $229 \pm 3(39.7 \pm 0.6 \%)$ \\
IT-4F & $626 \pm 18$ & $123 \pm 10(19.7 \pm 1.8 \%)$ \\
ITIC & $705 \pm 7$ & $127 \pm 3(18.1 \pm 0.3 \%)$ \\
\hline
\end{tabular}




\subsection{Network analysis}

We sought to quantify and characterize the clusters that are found in our MD simulations based on electronic couplings. In this framework, networks can be defined as a function of coupling threshold [57]. For a given electronic coupling threshold value $\left(\mathrm{V}_{\mathrm{T}}\right)$, we obtain the number and size of clusters for which the connectivity between molecules has electronic couplings larger than $\mathrm{V}_{\mathrm{T}}$. We note that a small threshold value naturally leads to a low number of networks with large sizes because connections can be formed more easily; in contrast, for a large threshold value, the clusters tend to contain small fragments since it is more difficult to establish the required extent of connection. For electrons, holes, and excitons, the number of networks increases more slowly in Y6 than ITIC-type acceptors as a function of threshold value (see Figures 3, S2, and S3). The maximum cluster sizes, in terms of the number of molecules the cluster contains, are also larger for Y6 than the ITIC-type acceptors. Taken together, these results indicate that the Y6 networks are extremely well-connected based on our MD morphologies. Examples of the top five largest clusters for electron, hole, and exciton networks are shown in Figures 3, S2, and S3. A simple visualization confirms that the clusters in Y6 are larger and better-connected than in ITIC-type acceptors. 

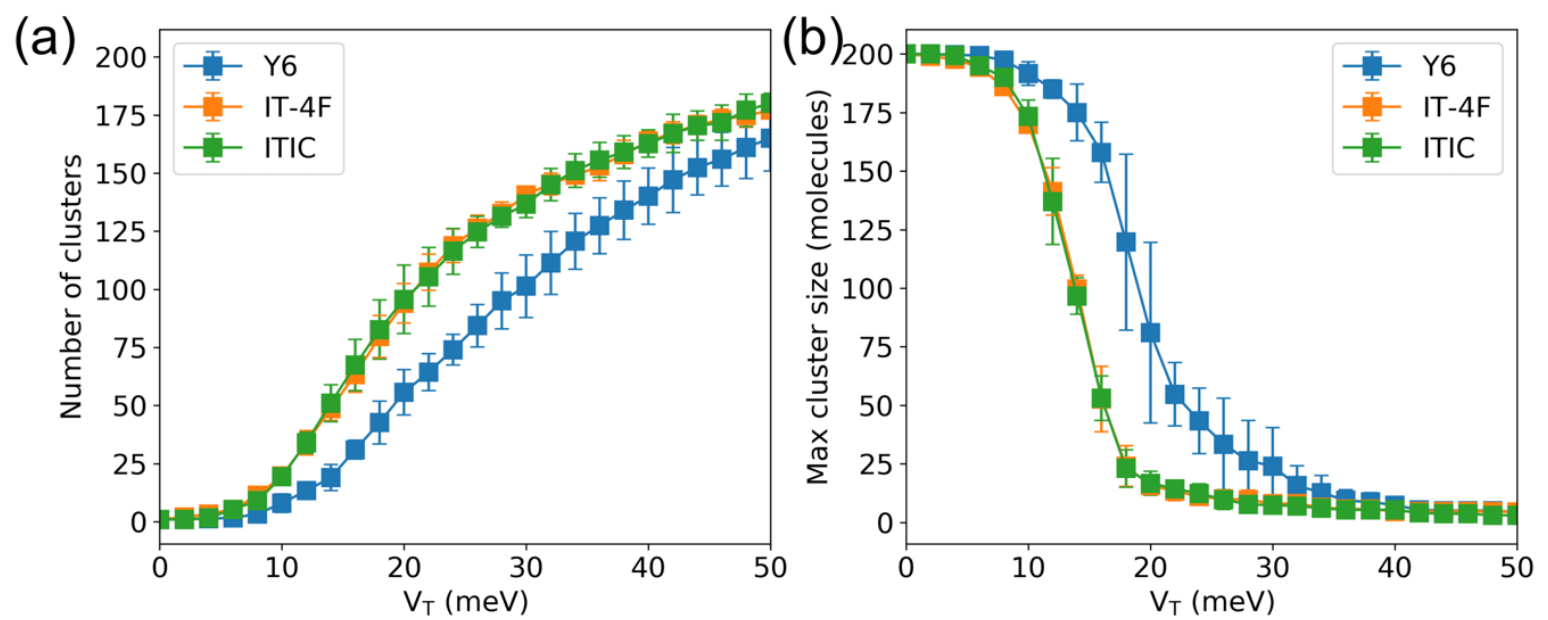

(c)

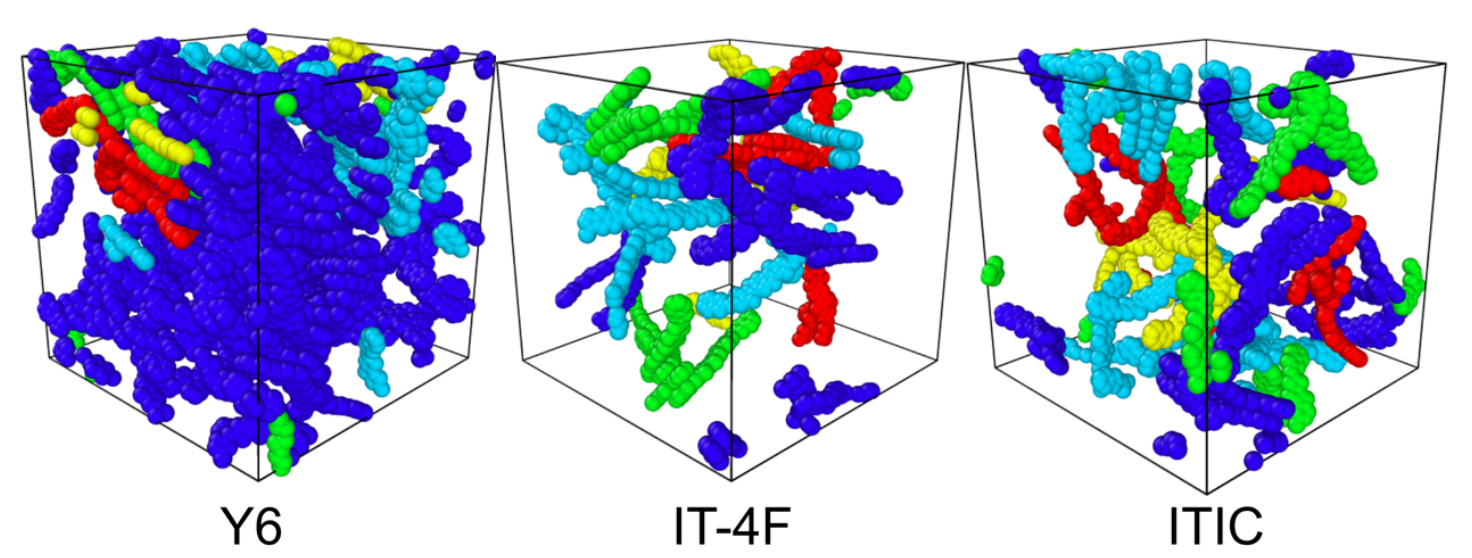

Figure 3. (a) Number of clusters and (b) maximum size versus threshold coupling $\left(\mathrm{V}_{\mathrm{T}}\right)$ for electron transport. (c) Illustration of the 5 largest clusters by color for electron transport at $\mathrm{V}_{\mathrm{T}}=20 \mathrm{meV}$.

\subsection{Charge and exciton transport rates}

In order to characterize the overall transport process, we calculated the rates between the dimers extracted from the MD simulations, based on the semi-classical Marcus equation. The distributions of the transfer rates are tabulated in Table 2. Our results indicate the remarkable property of the Y6 acceptor that it not only has fast electron transport rates, but also high populations of fast transfer rates for holes and excitons. In agreement with our calculations, experimental data have shown that Y6-based blends generally tend to possess superior electron transport properties based 
on mobility and diffusion data [58-61]. Our results also point to favorable hole transport in Y6, which supports the recent description of its ambipolar nature [33, 27, 25].

Table 2. Distribution (in \%) of electron, hole, and exciton transport rates. The brackets in the first row specify the results for electron $\left(\mathrm{r}_{\mathrm{e}}\right)$, hole $\left(\mathrm{r}_{\mathrm{h}}\right)$, and exciton $\left(\mathrm{r}_{\mathrm{x}}\right)$ rates.

\begin{tabular}{|c|c|c|c|c|c|c|c|c|c|}
\hline \multirow[b]{2}{*}{ Rate $\left(\mathrm{s}^{-1}\right)$} & \multicolumn{3}{|c|}{$\mathbf{r}_{\mathbf{e}}$} & \multicolumn{3}{|c|}{$\mathbf{r}_{\mathbf{h}}$} & \multicolumn{3}{|c|}{$\mathbf{r}_{\mathrm{x}}$} \\
\hline & Y6 & IT-4F & ITIC & Y6 & IT-4F & ITIC & Y6 & IT-4F & ITIC \\
\hline$>10^{12}$ & 54.2 & 53.3 & 47.8 & 47.3 & 47.4 & 44.4 & 86.3 & 81.7 & 77.2 \\
\hline$>10^{11}$ & 96.7 & 98.6 & 97.7 & 95.6 & 97.6 & 97.3 & 99.5 & 99.0 & 98.3 \\
\hline$>10^{10}$ & 100 & 100 & 100 & 100 & 100 & 100 & 100 & 100 & 100 \\
\hline
\end{tabular}

To understand the different factors that contribute to the observed rates, the role of reorganization energy and electronic couplings were investigated within the Marcus framework. The reorganization energy is related to the energetic penalty associated with structural changes between initial and final states. Our calculations indicate that, among the three NFAs, Y6 has surprisingly the highest reorganization energies for electron and hole transport, but the lowest reorganization energies associated with exciton transport (Table S1). The fact that Y6 consistently shows high population of large transport rates then means that the electronic couplings play a dominant role in controlling the rates. Our data indicate that $\mathrm{Y} 6$ has indeed higher electronic coupling values for electron, hole, and exciton (Table S2), which reflect a higher wavefunction overlap between adjacent molecules [47]. This is a consequence of the Y6 molecular architecture that enables molecular packing with highly overlapped backbones, which in this case results in large electronic couplings. As mentioned previously, these types of packings are prevented in the ITIC-type acceptors due to the presence of their bulky out-of-plane side-chains. 
The calculated transport rates can also be analyzed from the perspective of energetic disorder. Disorder can be defined as the standard deviation of the energetic distributions of the relevant states and is known to affect significantly the transport properties [47]. We recall that the total disorder $\left(\sigma_{\mathrm{T}}\right)$ can be decomposed into a dynamic component ( $\sigma_{\mathrm{D}}$, time-dependent; due to molecular vibrations) and a static component ( $\sigma_{\mathrm{s}}$, time-independent; due to lack of perfect order) as follows: $\sigma_{T}^{2}=\sigma_{D}^{2}+\sigma_{S}^{2}[62,63]$. From our data, among the three acceptors, Y6 has a relatively small total disorder for EA and IP (Table S3, Figures S4 and S5). The small total disorder for EA is related to the dynamic component, while the small total disorder for IP is due to both the dynamic and static terms. The small dynamic disorder can be related to the larger conformational rigidity of Y6, as discussed earlier. Our results are consistent with charge extraction measurements indicating that Y6 blends have one of the lowest energic disorder among non-fullerene acceptors [64].

Although our calculations point to remarkable exciton transport rates in Y6, recent experimental data do not provide such a clear-cut conclusion. From EQE spectra measured in a bilayer configuration, Firdaus et al. reported that the exciton diffusion length in Y6 is similar to that IT4F, while that in ITIC is significantly smaller [65]. From quantum-chemical calculations, these authors also found that $\mathrm{Y} 6$ has the lowest excited-state reorganization energy, followed by IT-4F and ITIC, which is consistent with our calculations (Table S1) and should in principle contribute to high exciton diffusion rates $[33,25,66]$. Recalling that the diffusion length is a function of mobility and lifetime, it is interesting to note that $\mathrm{Y} 6$ has also an extremely long exciton lifetime, at the origin of a highly efficient exciton dissociation process [67]. Another possible cause of this discrepancy could come from the structures derived from the MD simulations; while our present work only considers the amorphous regions of the acceptor domains within the actively layer, multiple domains with varying degrees of crystallinity are present in actual systems. 


\subsection{Dissecting the Y6 packing configurations}

Based on the morphologies coming out of the MD simulations, it is of interest to clarify the types of packing configurations present in the NFA films in order to assess the impact of the molecular arrangements on eventual performance. Here, we employed the k-means clustering algorithm to quantify the types of packing configurations that appear in the MD-simulated thin films (see details in SI). The distributions and types of Y6 configurations are shown in Figure 4. A signification portion of the configuration corresponds to terminal-terminal interactions (TT); it corresponds to $\sim 45 \%$ among all dimers extracted from the MD simulations of Y6. However, there also exist a few packing configurations that are very similar to those for dimers in the experimental crystal structures of Y6 and its derivatives [30, 26, 25, 27-29]. Specifically, there appear dimers with core/core and terminal/terminal interactions (CC-TT), or there can be dimers with core/terminal interactions involving different moieties (CT-CT), see Figure 4. These CC-TT and CT-CT configurations contribute to $20 \%$ of all dimers in the Y6 neat film (we note that while we mentioned this observation in our previous work [25], we did not quantify nor characterize these configurations in detail). For ITIC-type acceptors, the results based on similar analyses are

reported in the SI (Figures S7 and S8). The majority of ITIC-type acceptors interact via the TT configuration, which has been confirmed in many previous instances [56, 49, 68-71]. In the following sections, we focus on the TT, CC-TT, and CT-CT configurations of Y6 since they share structural similarity in both amorphous and crystalline phases. 

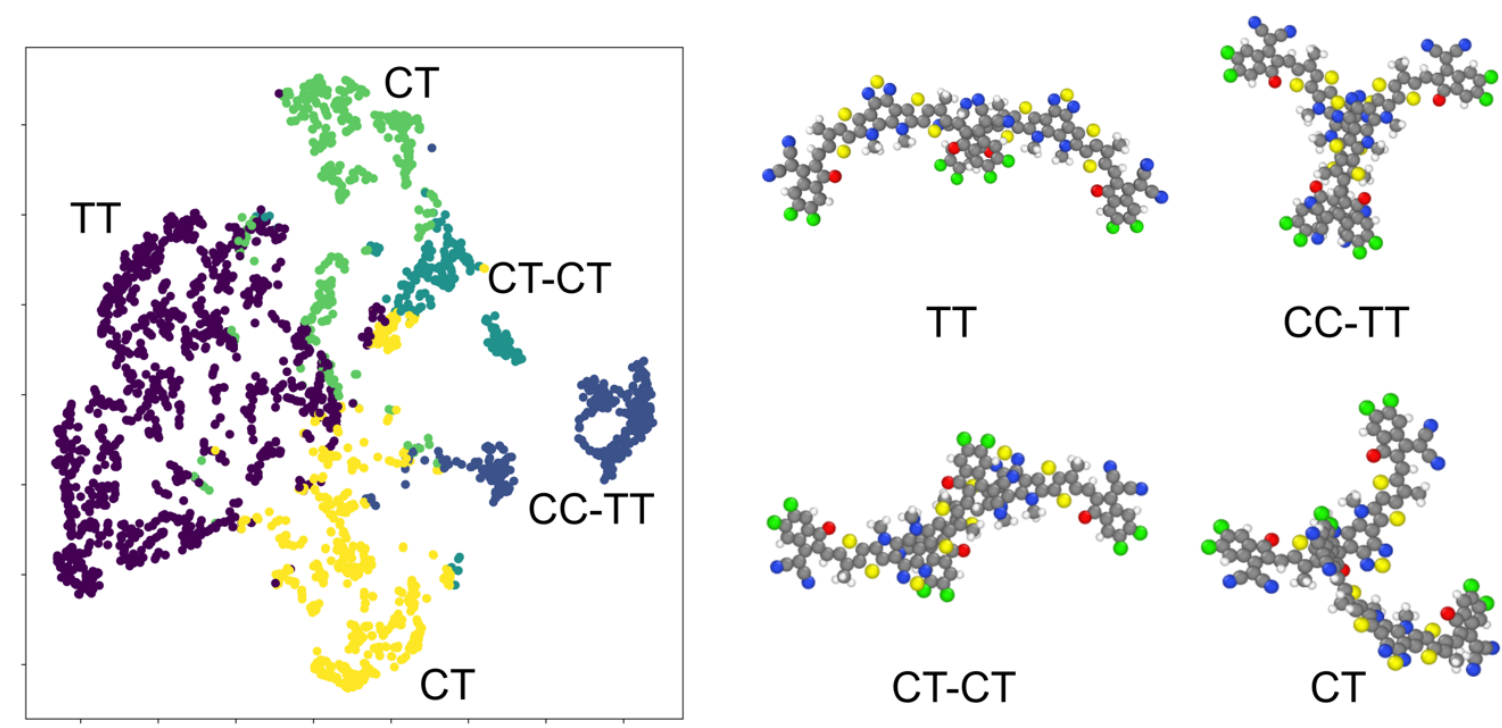

TT
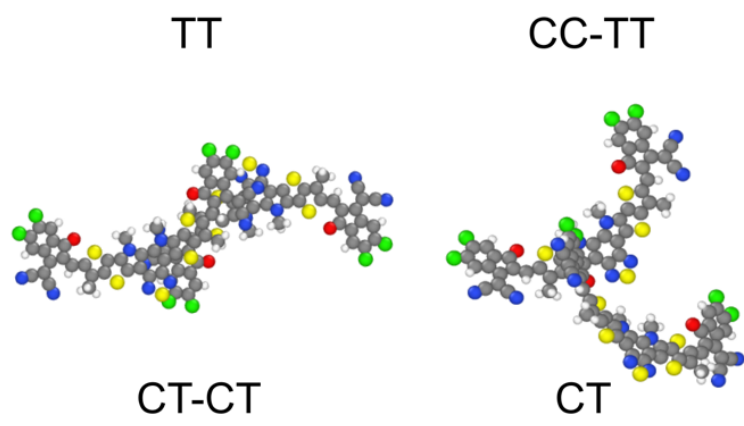

Figure 4. Illustration of the packing distributions and configurations typically found in Y6. The examples of configurations shown above were extracted directly from the MD simulations (the side chains were removed to improve clarity).

To identify the dominant interactions that govern the molecular packing, we performed SAPT0 calculations on 10 independent pairs for each configuration extracted directly from the MDsimulated films (Figure 5). The chosen configurations are shown in the SI (Figures S9 - S13). For Y6, the variations in packing configuration lead to different degrees of intermolecular interactions involving electrostatic, exchange-repulsion, induction, and dispersion terms. Relative to the TT configuration, the CC-TT and CT-CT configurations have a significantly higher degree of exchange-repulsion interactions and especially dispersion interactions, which overall leads to an increase in the magnitude of the total interaction energy and higher stabilization. The stronger interaction energies of the CC-TT and CT-CT configurations have implication on the aggregation of Y-type acceptors and explains their appearance in spin-coated films [25]. In comparison to the ITIC-type acceptors in which the TT configuration dominates, the TT configuration in Y6 has 
similar magnitudes of the electrostatics, exchange-repulsion, induction, and dispersion terms (Figure S14).

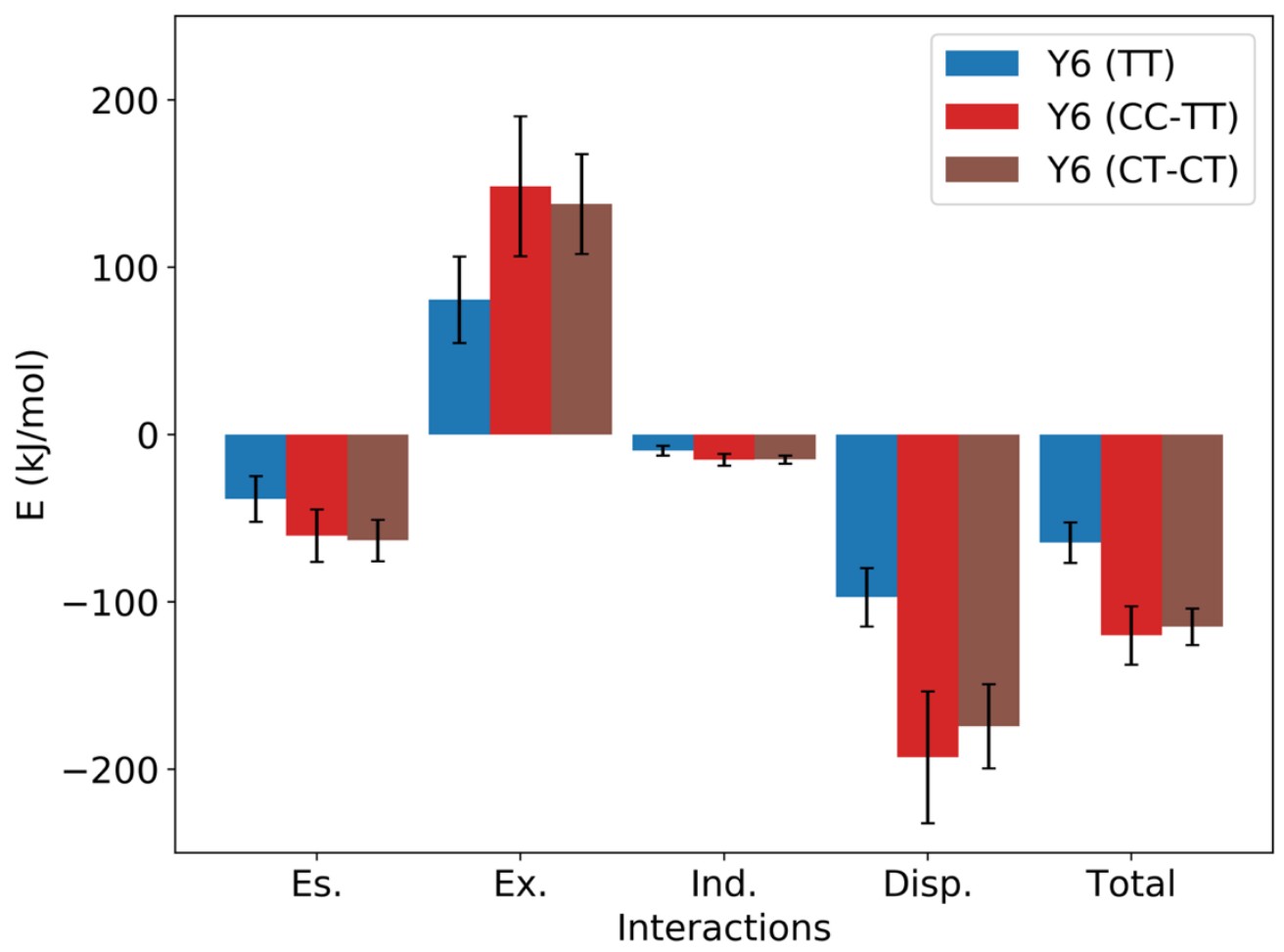

Figure 5. Decomposition of the molecular interactions using SAPT0 for the main Y6 configurations. The total interactions include electrostatic (Es.), exchange-repulsion (Ex.), induction (Ind.), and dispersion (Disp.) terms. The error bars represent in each case the standard deviation from 10 independent evaluations.

At this stage, it is useful to try and understand how these configurations can influence the electronic couplings and transport rates. When partitioning the electronic properties of Y6 based on its packing configurations, the CC-TT configuration is always found to lead to the highest coupling values for electron, hole, and exciton transport (Table 3). Besides the CC-TT configuration, the CT-CT configuration also appears to have desirable couplings for electron and hole transport, but 
not for exciton transport. All of these trends are reflected in the calculated rates for electron, hole, and exciton transport (Table S5). Thus, our result clearly indicate that the CC-TT and CT-CT configurations play a major role in enhancing the performance of Y6-based solar cells. The electronic couplings and transport rates as a function of packing configurations were also analyzed for the ITIC-type acceptors (Tables S4 and S5). In general, none of the configurations from ITICtype acceptors have electronic couplings or rates that surpass those of the best Y6 configurations.

Table 3. Average and maximum electronic couplings for LUMO-LUMO $\left(\mathrm{V}_{\mathrm{LL}}\right)$ and HOMOHOMO $\left(\mathrm{V}_{\mathrm{HH}}\right)$ interactions, and exciton energy transfer $\left(\mathrm{V}_{\mathrm{X}}\right)$, based on packing configurations found in the MD simulations. All values are in meV and maximum values are in parentheses.

\begin{tabular}{ccccc}
\hline & Config & $\mathbf{V}_{\mathbf{L L}}(\mathbf{m a x})$ & $\mathbf{V}_{\mathbf{H H}}(\mathbf{m a x})$ & $\mathbf{V}_{\mathbf{X}}(\mathbf{m a x})$ \\
\hline Y6 & TT & $20.4(106)$ & $12.6(41.4)$ & $36.0(91.6)$ \\
& CC-TT & $30.2(127)$ & $27.1(87.6)$ & $41.9(67.2)$ \\
& CT-CT & $22.8(81.4)$ & $23.0(77.1)$ & $18.8(60.6)$ \\
\hline
\end{tabular}

\section{Synopsis}

The Y6 non-fullerene acceptor and its derivatives have been responsible for the recent remarkable jump in the power conversion efficiencies of organic solar cells. It is thus of major interest to determine the factors that contribute to their greater efficacy with respect to the initial generation of NFAs involving ITIC and its derivatives. Here, we focused on the molecular-scale packing and the associated electronic properties in neat amorphous films of Y6, IT-4F, and ITIC, based on a combination of MD simulations and long-range corrected DFT calculations. Our main findings can be summarized as follows: 
- There are drastic differences in molecular packing between Y6 and ITIC-type films. The MD simulations highlight that Y6 has more directional molecular stacks and a higher number of face-on interactions. The reason is that Y6 does not carry the bulky out-of-plane side-chains, typical of ITIC-type acceptors, which allows better packing at its cores. Better packing is also promoted in Y6 via its higher molecular rigidity and therefore higher extent of co-planarity versus ITIC-type acceptors.

- Y6 clusters are more well-connected and extensive than in the ITIC-type acceptors, which points to better networks for charge and exciton transport.

- Y6 consistently displays higher populations of fast transport rates for electrons, holes, and excitons. The origin can be found mainly in larger electronic couplings, which is again related to the involvement of the $\mathrm{Y} 6$ cores in the packing configurations, thereby allowing greater wavefunction overlap. For exciton transport, Y6 also shows smaller excited-state reorganization energy relative to ITIC-type acceptors.

- The packing configurations involving the core moieties (CC-TT and CT-CT interactions), that are found in the Y6 amorphous films, are very similar to those of the dimers found in the Y6 single-crystal structure. The CC-TT configuration has the highest electronic couplings and transport rates for electrons, holes, and excitons among all configurations described in this work; the presence of such configurations is the result of favorable intermolecular interactions associated mainly with dispersion (induced dipole-induced dipole) dominated binding energies.

To conclude, our study has underlined fundamental differences between neat films of Y6 and ITIC-type acceptors and points to several factors why Y6 and its derivatives currently tend to be the leading acceptors in high-performance OSCs. For Y-type acceptors, the shape, moieties, side- 
chain structures and locations control the material's properties at multiple length scales including electronic properties and molecular packing.

\section{Data availability}

The data reported in this article are available upon request.

\section{Declaration of competing interest}

The authors declare no competing financial interest.

\section{Acknowledgments}

This work was by the Department of the Navy, Office of Naval Research (Award No. N00014-201-2110). We thank the DOD High Performance Computing Modernization Program as well as the Advanced Computing Environment (PACE) at the Georgia Institute of Technology for computational resources. The authors are most grateful to Dr. Veaceslav Coropceanu and Dr. Tonghui Wang for stimulating discussions. 


\section{References}

[1] G. Li, R. Zhu, Y. Yang, Polymer solar cells, Nat. Photon. 6 (2012), 153-161.

[2] L. Lu, T. Zheng, Q. Wu, A. M. Schneider, D. Zhao, L. Yu, Recent advances in bulk heterojunction polymer solar cells, Chem. Rev. 115 (2015), 12666-12731.

[3] A. Polman, M. Knight, E. C. Garnett, B. Ehrler, W. C. Sinke, Photovoltaic materials: present efficiencies and future challenges, Science 352 (2016), aad4424.

[4] O. Inganäs, Organic photovoltaics over three decades, Adv. Mater. 30 (2018), e1800388.

[5] Y. Lin, J. Wang, Z. G. Zhang, H. Bai, Y. Li, D. Zhu, X. Zhan, An electron acceptor challenging fullerenes for efficient polymer solar cells, Adv. Mater. 27 (2015), 1170-1174.

[6] F. Zhao, H. Zhang, R. Zhang, J. Yuan, D. He, Y. Zou, F. Gao, Emerging approaches in enhancing the efficiency and stability in non-fullerene organic solar cells, Adv. Energy Mater. 10 (2020), 2002746.

[7] A. Karki, A. J. Gillett, R. H. Friend, T.-Q. Nguyen, The path to 20\% power conversion efficiencies in nonfullerene acceptor organic solar cells, Adv. Energy Mater. (2020), 2003441.

[8] A. Armin, W. Li, O. J. Sandberg, Z. Xiao, L. Ding, J. Nelson, D. Neher, K. Vandewal, S. Shoaee, T. Wang, H. Ade, T. Heumüller, C. Brabec, P. Meredith, A history and perspective of non-fullerene electron acceptors for organic solar cells, Adv. Energy Mater. (2021), 2003570.

[9] C. Cui, Recent progress in fused-ring based nonfullerene acceptors for polymer solar cells, Front. Chem. 6 (2018).

[10] C. J. Brabec, S. Gowrisanker, J. J. M. Halls, D. Laird, S. Jia, S. P. Williams, Polymerfullerene bulk-heterojunction solar cells, Adv. Mater. 22 (2010), 3839-3856.

[11] C. Deibel, V. Dyakonov, Polymer-fullerene bulk heterojunction solar cells, Rep. Prog. Phys. 73 (2010), 096401.

[12] J. Nelson, Polymer: fullerene bulk heterojunction solar cells, Mater. Today 14 (2011), 462470.

[13] J. Zhao, Y. Li, G. Yang, K. Jiang, H. Lin, H. Ade, W. Ma, H. Yan, Efficient organic solar cells processed from hydrocarbon solvents, Nat. Energy 1 (2016), 15027.

[14] L. Feng, J. Yuan, Z. Zhang, H. Peng, Z.-G. Zhang, S. Xu, Y. Liu, Y. Li, Y. Zou, Thieno[3,2-b]pyrrolo-Fused Pentacyclic Benzotriazole-Based Acceptor for Efficient Organic Photovoltaics, ACS Appl. Mater. Inter. 9 (2017), 31985-31992.

[15] Q. Wei, W. Liu, M. Leclerc, J. Yuan, H. Chen, Y. Zou, A-DA'D-A non-fullerene acceptors for high-performance organic solar cells, Sci. China Chem. 63 (2020), 1352-1366.

[16] P. Cheng, Y. Yang, Narrowing the band gap: the key to high-performance organic photovoltaics, Acc. Chem. Res. 53 (2020), 1218-1228.

[17] J. Yuan, Y. Zhang, L. Zhou, G. Zhang, H. L. Yip, T. K. Lau, X. Lu, C. Zhu, H. Peng, P. A. Johnson, M. Leclerc, Y. Cao, J. Ulanski, Y. Li, Y. Zou, Single-junction organic solar cell with over $15 \%$ efficiency using fused-ring acceptor with electron-deficient core, Joule 3 (2019), 1140-1151.

[18] Q. Liu, Y. Jiang, K. Jin, J. Qin, J. Xu, W. Li, J. Xiong, J. Liu, Z. Xiao, K. Sun, S. Yang, X. Zhang, L. Ding, 18\% Efficiency organic solar cells, Sci. Bull. 65 (2020), 272-275.

[19] Y. Lin, Y. Firdaus, F. H. Isikgor, M. I. Nugraha, E. Yengel, G. T. Harrison, R. Hallani, A. El-Labban, H. Faber, C. Ma, X. Zheng, A. Subbiah, C. T. Howells, O. M. Bakr, I. McCulloch, S. De Wolf, L. Tsetseris, T. D. Anthopoulos, Self-assembled monolayer enables hole transport layer-free organic solar cells with $18 \%$ efficiency and improved operational stability, ACS Energy Lett. 5 (2020), 2935-2944. 
[20] S. Li, C.-Z. Li, M. Shi, H. Chen, New phase for organic solar cell researches: emergence of Y-series electron acceptors and their perspectives, ACS Energy Lett. 5 (2020), 1554-1567.

[21] R. Yu, G. Wu, Z. Tan, Realization of high performance for PM6:Y6 based organic photovoltaic cells, J. Energy Chem. 61 (2021), 29-46.

[22] Z.-C. Wen, H. Yin, X.-T. Hao, Recent progress of PM6:Y6-based high efficiency organic solar cells, Surf. Interfaces 23 (2021), 100921.

[23] Q. Guo, Q. Guo, Y. Geng, A. Tang, M. Zhang, M. Du, X. Sun, E. Zhou, Recent advances in PM6:Y6-based organic solar cells, Mater. Chem. Front. (2021).

[24] H. Lai, F. He, Crystal engineering in organic photovoltaic acceptors: a 3D network approach, Adv. Energy Mater. 10 (2020), 2002678.

[25] G. Zhang, X. K. Chen, J. Xiao, P. C. Y. Chow, M. Ren, G. Kupgan, X. Jiao, C. C. S. Chan, X. Du, R. Xia, Z. Chen, J. Yuan, Y. Zhang, S. Zhang, Y. Liu, Y. Zou, H. Yan, K. S. Wong, V. Coropceanu, N. Li, C. J. Brabec, J. L. Brédas, H. L. Yip, Y. Cao, Delocalization of exciton and electron wavefunction in non-fullerene acceptor molecules enables efficient organic solar cells, Nat. Commun. 11 (2020), 3943.

[26] L. Zhu, M. Zhang, G. Zhou, T. Hao, J. Xu, J. Wang, C. Qiu, N. Prine, J. Ali, W. Feng, X. Gu, Z. Ma, Z. Tang, H. Zhu, L. Ying, Y. Zhang, F. Liu, Efficient organic solar cell with $16.88 \%$ efficiency enabled by refined acceptor crystallization and morphology with improved charge transfer and transport properties, Adv. Energy Mater. 10 (2020), 1904234.

[27] C. Xiao, C. Li, F. Liu, L. Zhang, W. Li, Single-crystal field-effect transistors based on a fused-ring electron acceptor with high ambipolar mobilities, J. Mater. Chem. C 8 (2020), 53705374.

[28] W. Zhu, A. P. Spencer, S. Mukherjee, J. M. Alzola, V. K. Sangwan, S. H. Amsterdam, S. M. Swick, L. O. Jones, M. C. Heiber, A. A. Herzing, G. Li, C. L. Stern, D. M. DeLongchamp, K. L. Kohlstedt, M. C. Hersam, G. C. Schatz, M. R. Wasielewski, L. X. Chen, A. Facchetti, T. J. Marks, Crystallography, morphology, electronic structure, and transport in non-fullerene/nonindacenodithienothiophene polymer:Y6 solar cells, J. Am. Chem. Soc. 142 (2020), 14532-14547. [29] F. Lin, K. Jiang, W. Kaminsky, Z. Zhu, A. K. Jen, A non-fullerene acceptor with enhanced intermolecular pi-core interaction for high-performance organic solar cells, J. Am. Chem. Soc. 142 (2020), 15246-15251.

[30] H. Lai, Q. Zhao, Z. Chen, H. Chen, P. Chao, Y. Zhu, Y. Lang, N. Zhen, D. Mo, Y. Zhang, F. He, Trifluoromethylation enables a 3D interpenetrated low-band-gap acceptor for efficient organic solar cells, Joule 4 (2020), 688-700.

[31] T. Wang, G. Kupgan, J. L. Brédas, Organic photovoltaics: relating chemical structure, local morphology, and electronic properties, Trends Chem. 2 (2020), 535-554.

[32] Y. Cui, P. Zhu, X. Liao, Y. Chen, Recent advances of computational chemistry in organic solar cell research, J. Mater. Chem. C 8 (2020), 15920-15939.

[33] L. Perdigon-Toro, H. Zhang, A. Markina, J. Yuan, S. M. Hosseini, C. M. Wolff, G. Zuo, M. Stolterfoht, Y. Zou, F. Gao, D. Andrienko, S. Shoaee, D. Neher, Barrierless free charge generation in the high-performance PM6:Y6 bulk heterojunction non-fullerene solar cell, Adv. Mater. (2020), 1906763.

[34] Z. Tu, G. Han, Y. Yi, Barrier-free charge separation enabled by electronic polarization in high-efficiency non-fullerene organic solar cells, J. Phys. Chem. Lett. 11 (2020), 2585-2591.

[35] G. Han, T. Hu, Y. Yi, Reducing the singlet-triplet energy gap by end-group pi-pi stacking toward high-efficiency organic photovoltaics, Adv Mater 32 (2020), e2000975. 
[36] Z. Cao, S. Yang, B. Wang, X. Shen, G. Han, Y. Yi, Multi-channel exciton dissociation in D18/Y6 complexes for high-efficiency organic photovoltaics, J. Mater. Chem. A 8 (2020), 20408-20413.

[37] S. Plimpton, Fast parallel algorithms for short-range molecular-dynamics, J. Comput. Phys. 117 (1995), 1-19.

[38] W. L. Jorgensen, J. Tirado-Rives, The OPLS potential functions for proteins - energy minimizations for crystals of cyclic-peptides and crambin, J. Am. Chem. Soc. 110 (1988), 16571666.

[39] M. K. Dahlgren, P. Schyman, J. Tirado-Rives, W. L. Jorgensen, Characterization of biaryl torsional energetics and its treatment in OPLS all-atom force fields, J. Chem. Inf. Model. 53 (2013), 1191-1199.

[40] N. E. Jackson, K. L. Kohlstedt, B. M. Savoie, M. O. de la Cruz, G. C. Schatz, L. X. Chen, M. A. Ratner, Conformational order in aggregates of conjugated polymers, J. Am. Chem. Soc. 137 (2015), 6254-6262.

[41] J. D. Chai, M. Head-Gordon, Long-range corrected hybrid density functionals with damped atom-atom dispersion corrections, Phys. Chem. Chem. Phys. 10 (2008), 6615-6620.

[42] D. A. Case, I. Y. Ben-Shalom, S. R. Brozell, D. S. Cerutti, I. T.E. Cheatham, V. W. D. Cruzeiro, T. A. Darden, R. E. Duke, D. Ghoreishi, M. K. Gilson, H. Gohlke, A. W. Goetz, D. Greene, R. Harris, N. Homeyer, S. Izadi, A. Kovalenko, T. Kurtzman, T. S. Lee, S. LeGrand, P. Li, C. Lin, J. Liu, T. Luchko, R. Luo, D. J. Mermelstein, K. M. Merz, Y. Miao, G. Monard, C. Nguyen, H. Nguyen, I. Omelyan, A. Onufriev, F. Pan, R. Qi, D. R. Roe, A. Roitberg, C. Sagui, S. Schott-Verdugo, J. Shen, C. L. Simmerling, J. Smith, R. Salomon-Ferrer, J. Swails, R. C. Walker, J. Wang, H. Wei, R. M. Wolf, X. Wu, L. Xiao, D. M. York, P. A. Kollman AMBER 2018, University of California, San Francisco, 2018.

[43] M. J. Frisch, G. W. Trucks, H. B. Schlegel, G. E. Scuseria, M. A. Robb, J. R. Cheeseman, G. Scalmani, V. Barone, G. A. Petersson, H. Nakatsuji, X. Li, M. Caricato, A. V. Marenich, J. Bloino, B. G. Janesko, R. Gomperts, B. Mennucci, H. P. Hratchian, J. V. Ortiz, A. F. Izmaylov, J. L. Sonnenberg, Williams, F. Ding, F. Lipparini, F. Egidi, J. Goings, B. Peng, A. Petrone, T. Henderson, D. Ranasinghe, V. G. Zakrzewski, J. Gao, N. Rega, G. Zheng, W. Liang, M. Hada, M. Ehara, K. Toyota, R. Fukuda, J. Hasegawa, M. Ishida, T. Nakajima, Y. Honda, O. Kitao, H. Nakai, T. Vreven, K. Throssell, J. A. Montgomery Jr., J. E. Peralta, F. Ogliaro, M. J. Bearpark, J. J. Heyd, E. N. Brothers, K. N. Kudin, V. N. Staroverov, T. A. Keith, R. Kobayashi, J. Normand, K. Raghavachari, A. P. Rendell, J. C. Burant, S. S. Iyengar, J. Tomasi, M. Cossi, J. M. Millam, M. Klene, C. Adamo, R. Cammi, J. W. Ochterski, R. L. Martin, K. Morokuma, O. Farkas, J. B. Foresman, D. J. Fox Gaussian 16 Rev. C.01, Wallingford, CT, 2016.

[44] L. J. Abbott, K. E. Hart, C. M. Colina, Polymatic: a generalized simulated polymerization algorithm for amorphous polymers, Theor. Chem. Acc. 132 (2013), 1334.

[45] M. E. Fortunato, C. M. Colina, pysimm: a python package for simulation of molecular systems, SoftwareX 6 (2017), 7-12.

[46] L. Kronik, S. Kümmel, Dielectric screening meets optimally tuned density functionals, Adv. Mater. 30 (2018), 1706560.

[47] V. Coropceanu, J. Cornil, D. A. da Silva Filho, Y. Olivier, R. Silbey, J. L. Brédas, Charge transport in organic semiconductors, Chem. Rev. 107 (2007), 926-952.

[48] R. A. Marcus, Electron-transfer reactions in chemistry. Theory and experiment, Rev. Mod. Phys. 65 (1993), 599-610. 
[49] T. Wang, J. L. Brédas, Nonfullerene small-molecule acceptors for organic photovoltaics: understanding the impact of methoxy substitution position on molecular packing and electrontransfer properties, Adv. Funct. Mater. 29 (2019), 1806845.

[50] T. Wang, J. L. Brédas, Organic solar cells based on non-fullerene small-molecule acceptors: impact of substituent position, Matter 2 (2020), 119-135.

[51] R. M. Parrish, L. A. Burns, D. G. A. Smith, A. C. Simmonett, A. E. DePrince, E. G. Hohenstein, U. Bozkaya, A. Y. Sokolov, R. Di Remigio, R. M. Richard, J. F. Gonthier, A. M. James, H. R. McAlexander, A. Kumar, M. Saitow, X. Wang, B. P. Pritchard, V. Prakash, H. F. Schaefer, K. Patkowski, R. A. King, E. F. Valeev, F. A. Evangelista, J. M. Turney, T. D. Crawford, C. D. Sherrill, PSI4 1.1: an open-source electronic structure program emphasizing automation, advanced libraries, and interoperability, J. Chem. Theory Comput. 13 (2017), 31853197.

[52] B. Jeziorski, R. Moszynski, K. Szalewicz, Perturbation-theory approach to intermolecular potential-energy surfaces of van-der-waals complexes, Chem. Rev. 94 (1994), 1887-1930.

[53] M. K. Ravva, T. Wang, J. L. Brédas, Nature of the binding interactions between conjugated polymer chains and fullerenes in bulk heterojunction organic solar cells, Chem. Mater. 28 (2016), 8181-8189.

[54] J. L. Brédas, J. P. Calbert, D. A. da Silva Filho, J. Cornil, Organic semiconductors: a theoretical characterization of the basic parameters governing charge transport, Proc. Natl. Acad. Sci. USA 99 (2002), 5804-9.

[55] G. Han, Y. Yi, Z. Shuai, From molecular packing structures to electronic processes: theoretical simulations for organic solar cells, Adv. Energy Mater. 8 (2018), 1702743.

[56] G. Han, Y. Guo, X. Song, Y. Wang, Y. Yi, Terminal pi-pi stacking determines threedimensional molecular packing and isotropic charge transport in an A-pi-A electron acceptor for non-fullerene organic solar cells, J. Mater. Chem. C 5 (2017), 4852-4857.

[57] B. M. Savoie, K. L. Kohlstedt, N. E. Jackson, L. X. Chen, M. O. de la Cruz, G. C. Schatz, T. J. Marks, M. A. Ratner, Mesoscale molecular network formation in amorphous organic materials, Proc. Natl. Acad. Sci. USA 111 (2014), 10055-10060.

[58] K. Li, Y. Wu, Y. Tang, M. A. Pan, W. Ma, H. Fu, C. Zhan, J. Yao, Ternary blended fullerene-free polymer solar cells with $16.5 \%$ efficiency enabled with a higher-lumo-level acceptor to improve film morphology, Adv. Energy Mater. 9 (2019), 1901728.

[59] M. A. Pan, T. K. Lau, Y. Tang, Y. C. Wu, T. Liu, K. Li, M. C. Chen, X. Lu, W. Ma, C. Zhan, 16.7\%-efficiency ternary blended organic photovoltaic cells with PCBM as the acceptor additive to increase the open-circuit voltage and phase purity, J. Mater. Chem. A 7 (2019), 2071320722.

[60] Y. Wang, Q. Fan, X. Guo, W. Li, B. Guo, W. Su, X. Ou, M. Zhang, High-performance nonfullerene polymer solar cells based on a fluorinated wide bandgap copolymer with a high opencircuit voltage of 1.04 V, J. Mater. Chem. A 5 (2017), 22180-22185.

[61] N. Tokmoldin, S. M. Hosseini, M. Raoufi, L. Phuong, O. J. Sandberg, H. Guan, Y. Zou, D. Neher, S. Shoaee, Extraordinarily long diffusion length in PM6:Y6 organic solar cells, J. Mater. Chem. A 8 (2020), 7854-7860.

[62] N. R. Tummala, Z. L. Zheng, S. G. Aziz, V. Coropceanu, J. L. Brédas, Static and dynamic energetic disorders in the C60, PC61BM, C70, and PC71BM fullerenes, J. Phys. Chem. Lett. 6 (2015), 3657-3662.

[63] G. Kupgan, X. K. Chen, J. L. Brédas, Low energetic disorder in small-molecule nonfullerene electron acceptors, ACS Materials Lett. 1 (2019), 350-353. 
[64] J. Wu, J. Lee, Y.-C. Chin, H. Yao, H. Cha, J. Luke, J. Hou, J.-S. Kim, J. R. Durrant, Exceptionally low charge trapping enables highly efficient organic bulk heterojunction solar cells, Energy Environ. Sci. 13 (2020), 2422-2430.

[65] Y. Firdaus, V. M. Le Corre, S. Karuthedath, W. Liu, A. Markina, W. Huang, S. Chattopadhyay, M. M. Nahid, M. I. Nugraha, Y. Lin, A. Seitkhan, A. Basu, W. Zhang, I. McCulloch, H. Ade, J. Labram, F. Laquai, D. Andrienko, L. J. A. Koster, T. D. Anthopoulos, Long-range exciton diffusion in molecular non-fullerene acceptors, Nat. Commun. 11 (2020), 5220 .

[66] S. Chandrabose, K. Chen, A. J. Barker, J. J. Sutton, S. K. K. Prasad, J. Zhu, J. Zhou, K. C. Gordon, Z. Xie, X. Zhan, J. M. Hodgkiss, High exciton diffusion coefficients in fused ring electron acceptor films, J. Am. Chem. Soc. 141 (2019), 6922-6929.

[67] A. Classen, C. L. Chochos, L. Lüer, V. G. Gregoriou, J. Wortmann, A. Osvet, K. Forberich, I. McCulloch, T. Heumüller, C. J. Brabec, The role of exciton lifetime for charge generation in organic solar cells at negligible energy-level offsets, Nat. Energy 5 (2020), 711-719. [68] X. Shi, L. Zuo, S. B. Jo, K. Gao, F. Lin, F. Liu, A. K. Y. Jen, Design of a highly crystalline low-band gap fused-ring electron acceptor for high-efficiency solar cells with low energy loss, Chem. Mater. 29 (2017), 8369-8376.

[69] J. Mai, Y. Xiao, G. Zhou, J. Wang, J. Zhu, N. Zhao, X. Zhan, X. Lu, Hidden structure ordering along backbone of fused-ring electron acceptors enhanced by ternary bulk heterojunction, Adv. Mater. 30 (2018), 1802888.

[70] K. Jin, C. Deng, L. Zhang, D. Li, T. Li, F. Wang, Y. Yuan, Z. Xiao, L. Ding, A heptacyclic carbon-oxygen-bridged ladder-type building block for A-D-A acceptors, Mater. Chem. Front. 2 (2018), 1716-1719.

[71] X. Shi, X. Liao, K. Gao, L. Zuo, J. Chen, J. Zhao, F. Liu, Y. Chen, A. K. Y. Jen, An electron acceptor with broad visible-NIR absorption and unique solid state packing for as-cast high performance binary organic solar cells, Adv. Funct. Mater. 28 (2018), 1802324. 